\title{
Aproximaciones a un comportamiento alimentario saludable en estudiantes de la Universidad Nacional de Trujillo (Perú) Filial Huamachuco
}

\section{Approaches to a healthy eating behavior in students of the National University of Trujillo (Peru) Huamachuco Campus}

Dr. Rodolfo Moisés Vegas Niño ${ }^{1 *}$, Ing. Eresvita Carranza Rios ${ }^{1}$, Dra. Yolanda Fabiola \& Márquez Sandoval ${ }^{2}$

${ }^{\prime}$ Escuela de Ingeniería Agroindustrial, Universidad Nacional de Trujillo-Filial Huamachuco. Perú

${ }^{2}$ Centro Universitario de Ciencias de la Salud, Universidad de Guadalajara. Jalisco. México.

Correo electrónico:rvegas@unitru.edu.pe

\section{Resumen}

La alimentación influye en la salud, está debe contener los nutrientes esenciales, además de ser variada, adecuada e inocua. El objetivo de esta investigación descriptiva fue determinar las características de comportamiento alimentario saludable en estudiantes de la Universidad Nacional de Trujillo-Filial Huamachuco (Perú). Se aplicó un cuestionario a 318 estudiantes seleccionados de forma aleatoria de las carreras de Ingeniería Agroindustrial, Ingeniería de Minas, Administración y Enfermería. Los resultados evidencian que el 53.8\% de los encuestados consideraron como factor de elección de un alimento su contenido nutrimental. Un 33\% no lee las etiquetas nutrimentales por "falta de tiempo" y un $36.8 \%$ las lee, pero no las entiende. Un $46.2 \%$ no consume un alimento bajo el criterio "que no le gusta". Un $45.3 \%$ considera al vapor o hervido como la preparación más habitual de un alimento, siendo la madre en un $46.2 \%$ quien lo realiza. Un $66 \%$ considera tres ingestas diarias tanto entre semana como los fines de semana (sábado y domingo). Existe una mayor preferencia por el pollo y la carne de res frente al pescado. El 75.5\% considera una sopa o caldo en el almuerzo, asimismo un 58.8\% con una bebida dulce. El 39\% de los encuestados considera que les falta mayor motivación para mejorar su alimentación, existiendo un $10.1 \%$ que se muestran reacios a recibir consejos de nutrición. En gran medida los estudiantes encuestados presentan comportamientos saludables teniendo limitaciones en la interpretación de los valores nutricionales de las etiquetas lo que se recomienda que instituciones del Estado genere políticas públicas al respecto a través de programas de motivación y compromiso personal.

Palabras clave: alimentación, comportamiento, universitarios, hábitos. 


\begin{abstract}
Food influences health, it must contain essential nutrients, in addition to being varied, adequate and safe. The objective of this descriptive research was to determine the characteristics of healthy eating behavior in students of the National University of Trujillo-Subsidiary Huamachuco (Peru). A questionnaire was applied to 318 randomly selected students from the Agroindustrial Engineering, Mining Engineering, Administration and Nursing careers. The results show that $53.8 \%$ of the respondents considered its nutritional content as a factor in choosing a food. 33\% do not read nutritional labels due to "lack of time" and 36.8\% read them, but do not understand them. $46.2 \%$ do not consume a food under the criterion "that they do not like". $45.3 \%$ consider steamed or boiled as the most common preparation of food, with $46.2 \%$ being the mother who does it. $66 \%$ consider three daily intakes both during the week and on weekends (Saturday and Sunday). There is a greater preference for chicken and beef over fish. 75.5\% consider a soup or broth at lunch, likewise 58.8\% with a sweet drink. 39\% of those surveyed consider that they lack more motivation to improve their diet, while $10.1 \%$ are reluctant to receive nutrition advice. To a great extent, the students surveyed present healthy behaviors having limitations in the interpretation of the nutritional values of the labels, which is recommended that State institutions generate public policies in this regard through motivation programs and personal commitment.
\end{abstract}

Keywords: feeding, behavior, university students, habits.

\title{
Introducción
}

La alimentación saludable es considerada uno de los factores de promoción de una buena salud a lo largo de la vida. Una alimentación inadecuada es uno de los causantes de aparición de enfermedades no transmisibles (ENT), como diabetes, cáncer o enfermedades cardiovasculares (OMS, 2021). Se ha comprobado una correlación directa entre la dieta alimentaria y el peligro para desarrollar ENT. El modelo de una alimentación saludable contribuye a un excelente estado nutricional y a un estándar de vida en los seres humanos (Mariño et al., 2016).

Una dieta equilibrada comprende un aporte de nutrientes tanto de forma cuantitativa como cualitativa según las necesidades de la persona. La Organización Mundial de la Salud (1998) recomienda que un 50-55\% del total del aporte energético provenga de los carbohidratos, un $12-15 \%$ de las proteínas y un $30-35 \%$ de las grasas. Una alimentación variada debe incorporar alimentos de todos los grupos y en porciones adecuadas que permitan coberturar las necesidades energéticas y nutritivas que el cuerpo requiere (Cabrera y Galarza, 2008).

Caswell y Padberg (1992) consideran que, en la toma de decisiones referido a la elección de los alimentos, ésta puede estar influida, ya sea por la propaganda mayoritariamente televisiva, por recomendaciones para su ingesta o, en general, por la 
influencia de educación alimentaria llevado a cabo por el gobierno o por profesionales de la salud. El Consejo Nacional de Radio y Televisión del Perú publicó un informe en el que el 54\% de los avisos publicitarios en Lima referente a alimentos no son saludables (Consejo Consultivo de Radio y Televisión, 2012).

La juventud es una etapa decisiva en el desarrollo de la persona en la que se van adquiriendo hábitos que en la mayoría de los casos se mantienen en la edad adulta, con un riesgo o beneficio para la salud. Estos hábitos de riesgo incrementan la probabilidad de desarrollar ciertas patologías en la adultez, tales como arterosclerosis, trastornos del comportamiento alimentario, obesidad, diabetes y osteoporosis (Irazusta et al., 2007).

En nuestro país se han realizado estudios para determinar el conocimiento, actitudes y prácticas sobre alimentación saludable en adolescentes (Ruiz, 2019), a nivel universitario se evaluó el conocimiento sobre alimentación saludable en estudiantes de enfermería (Huanca y Valero, 2020), los factores que determinan los hábitos alimentarios (Chota y Simón, 2020), así como el estado nutricional y su relación con los hábitos alimenticios (Álvarez y Bendezú, 2011).

No se ha realizado estudios sobre el consumo y comportamiento alimentario en universitarios en Huamachuco-La Libertad (Perú). Por ello, se considera importante llevar a cabo esta investigación, de modo que, autoridades sanitarias, municipales y/o académicas en base a un sustento científico propongan políticas públicas que impulsen y difundan pautas de alimentación saludable que conlleven a la consecución de los Objetivos de Desarrollo Sostenible (ODS) al año 2030.

El objetivo de esta investigación fue determinar las características de comportamiento alimentario saludable en estudiantes de la Universidad Nacional de Trujillo-Filial Huamachuco (Perú). Teniendo como objetivos específicos: determinar el criterio de elección y no elección de un alimento para su consumo, así como el tipo de preparación más habitual de un alimento y la persona quien lo realiza.

\section{Método}

Se aplicó el método descriptivo, utilizando como instrumento de evaluación un cuestionario validado (Márquez et al., 2014) el cual fue aplicado de forma presencial en las aulas universitarias a 318 estudiantes de una población de 800 . La muestra fue determinada por ecuación para una población finita con un nivel de significancia del 5\% siendo la elección de los estudiantes de forma aleatoria simple.

Como criterios de inclusión se cuenta: Que el cuestionario se aplicó a los participantes de manera voluntaria, con consentimiento informado, respetando el anonimato de las respuestas. Se les formuló las mismas preguntas y en la misma secuencia, para obtener así la máxima fiabilidad en la información.

La población encuestada corresponde a los estudiantes matriculados en el semestre 2019-II (setiembre-diciembre 2019) y fue distribuida entre las carreras de Ingeniería Agroindustrial (75), Ingeniería de Minas (84), Enfermería (78) y 
Administración (81). No hubo distinción de género y las edades estuvieron comprendidas entre 18-26 años.

Como criterios de exclusión se cuenta: Estudiantes que no deseen participar en el estudio o que sigan algún régimen de alimentación (restricción dietética por enfermedad, prohibición dietética por religión), estudiantes en estado de gestación, periodo de lactancia o que presenten algún tipo de alergia alimentaria.

\section{Resultados}

El cuestionario aborda preguntas relacionadas a los criterios de elección de alimentos, hábitos de consumo, frecuencia y aceptabilidad de los mismos. En la Tabla 1 se muestra algunos comportamientos de consumo alimentario por parte de los estudiantes universitarios.

Entre las respuestas más destacadas se describe que el 33\% de los encuestados no lee las etiquetas por "falta de tiempo" (pregunta 2). Un $46.2 \%$ evita el consumo de algún alimento "porque no le gusta" (pregunta 3). Un 78.9\% cuando se siente satisfecho "deja de comer", sin embargo, el 12.9\% "sigue comiendo sin problema" (pregunta 6). Por otra parte, el $45.6 \%$ considera que su dieta alimentaria es "diferente solo algunas veces durante la semana" (pregunta 28). 


\section{Tabla 1}

Distribución porcentual de las respuestas emitidas en el cuestionario

\begin{tabular}{|c|c|c|c|c|c|c|c|}
\hline \multirow[t]{2}{*}{ Número de pregunta según cuestionario } & \multicolumn{7}{|c|}{ Alternativa (\%) } \\
\hline & a & b & c & d & e & f & $\mathbf{g}$ \\
\hline $\begin{array}{l}\text { 1. ¿Qué factor consideras más importante al } \\
\text { elegir un alimento para su consumo? }\end{array}$ & 28.3 & 4.7 & 3.8 & 9.4 & 53.8 & - & - \\
\hline $\begin{array}{l}\text { 2. Me es difícil leer las etiquetas } \\
\text { nutrimentales }\end{array}$ & 33.0 & 1.0 & 6.6 & 22.6 & 36.8 & - & - \\
\hline $\begin{array}{l}\text { 3. Si evitas algún alimento, ¿Por qué motivo } \\
\text { lo haces? }\end{array}$ & 46.2 & 31.1 & 15.1 & 7.6 & - & - & - \\
\hline $\begin{array}{l}\text { 4. ¿Cuál es la preparación más habitual de } \\
\text { tus alimentos? }\end{array}$ & 13.2 & 45.3 & 1.9 & 0.9 & 38.7 & - & - \\
\hline $\begin{array}{l}\text { 5. ¿Quién prepara tus alimentos con mayor } \\
\text { frecuencia durante la semana? }\end{array}$ & 31.1 & 46.2 & 12.3 & 10.4 & - & - & - \\
\hline $\begin{array}{l}\text { 6. ¿Qué haces normalmente cuando te } \\
\text { sientes satisfecho? }\end{array}$ & 78.9 & 4.4 & 12.9 & 3.8 & - & - & - \\
\hline $\begin{array}{l}\text { 7. ¿Qué haces con la grasa visible de la } \\
\text { carne? }\end{array}$ & 53.8 & 30.2 & 12.2 & 3.8 & - & - & - \\
\hline $\begin{array}{l}\text { 8. Habitualmente mastico cada bocado más } \\
\text { de } 25 \text { veces. }\end{array}$ & 5.0 & 25.2 & 35.9 & 29.2 & 4.7 & - & - \\
\hline $\begin{array}{l}\text { 21. ¿Qué sueles beber en mayor cantidad } \\
\text { durante el día? }\end{array}$ & 8.5 & 70.8 & 17.0 & 0.0 & 3.7 & - & - \\
\hline $\begin{array}{l}\text { 22. ¿Qué sueles ingerir habitualmente entre } \\
\text { comidas? }\end{array}$ & 6.3 & 58.5 & 8.5 & 2.2 & 12.3 & 3.7 & 8.5 \\
\hline $\begin{array}{l}\text { 24. ¿Con que frecuencia comes alimentos } \\
\text { fuera de casa? }\end{array}$ & 9.5 & 19.8 & 10.4 & 22.6 & 16.0 & 13.2 & 8.5 \\
\hline $\begin{array}{l}25 . \text { ¿Con qué frecuencia crees que comes } \\
\text { alimentos en exceso? }\end{array}$ & 3.8 & 9.4 & 17.9 & 33.0 & 12.3 & 12.3 & 11.3 \\
\hline $\begin{array}{l}\text { 26. ¿Qué haces o estarías dispuesto a hacer } \\
\text { para cuidar tu cuerpo? }\end{array}$ & 35.8 & 3.5 & 17.0 & 41.5 & 1.3 & 0.9 & - \\
\hline $\begin{array}{l}\text { 27. ¿Qué consideras que te hace falta para } \\
\text { mejorar tu alimentación? }\end{array}$ & 26.4 & 3.1 & 17.9 & 39.0 & 10.4 & 1.9 & 1.3 \\
\hline $\begin{array}{l}\text { 28. Consideras que tu dieta alimentaria (lo } \\
\text { que comes) es: }\end{array}$ & 40.6 & 45.6 & 8.5 & 5.3 & - & - & - \\
\hline $\begin{array}{l}\text { 29. ¿Crees que eres capaz de utilizar un } \\
\text { consejo de nutrición para mejorar tu estado } \\
\text { de salud? }\end{array}$ & 8.8 & 1.3 & 6.9 & 48.1 & 34.9 & - & - \\
\hline
\end{tabular}

Nota: a,b,c,d,e,f,g constituyen las alternativas de respuestas que se encuentran en el cuestionario del comportamiento alimentario. Hay preguntas que tiene 4 alternativas de respuesta y otras hasta 7 .

En la Tabla 2 se muestra la representación porcentual del nivel de preferencia (desde "me agrada mucho" hasta "me desagrada mucho") a diversos alimentos por parte de los estudiantes encuestados. Las frutas tienen un mayor grado de aceptabilidad en comparación a las verduras. Dentro del grupo de carbohidratos existe una mayor aceptabilidad por los "frijoles, garbanzos y lentejas" en comparación al "pan, papa, fideos y cereales". 


\section{Tabla 2}

Porcentaje (\%) de estudiantes universitarios que manifiestan su nivel de agrado/ desagrado frente a diversos alimentos

\begin{tabular}{lccccc}
\hline & $\begin{array}{c}\text { Me } \\
\text { agrada } \\
\text { mucho }\end{array}$ & $\begin{array}{c}\text { Me } \\
\text { Iftem }\end{array}$ & $\begin{array}{c}\text { Ni me } \\
\text { agrada ni } \\
\text { me } \\
\text { desagrada }\end{array}$ & $\begin{array}{c}\text { Me } \\
\text { desagrada }\end{array}$ & $\begin{array}{c}\text { Me } \\
\text { desagrada } \\
\text { mucho }\end{array}$ \\
\hline Frutas & 72.6 & 23.6 & 2.8 & 0.0 & 0.9 \\
Verduras & 28.3 & 50.0 & 18.9 & 1.3 & 1.6 \\
Carnes y pollo & 30.8 & 54.4 & 11.3 & 0.6 & 2.8 \\
$\begin{array}{l}\text { Pescados y mariscos } \\
\text { Lácteos }\end{array}$ & 34.0 & 43.4 & 16.0 & 3.5 & 3.1 \\
$\begin{array}{l}\text { Pan, papa, fideos, } \\
\text { cereales }\end{array}$ & 21.7 & 48.4 & 18.6 & 10.4 & 0.9 \\
$\begin{array}{l}\text { Frijoles, garbanzos, } \\
\text { lentejas }\end{array}$ & 12.9 & 51.6 & 30.8 & 2.8 & 1.9 \\
$\begin{array}{l}\text { Huevo } \\
\text { Almendras, nueces, }\end{array}$ & 31.1 & 49.1 & 15.4 & 1.9 & 2.5 \\
pasas, guindones & 29.2 & 43.4 & 21.7 & 3.8 & 1.9 \\
$\begin{array}{l}\text { Bebidas alcohólicas } \\
\text { Alimentos envasados }\end{array}$ & 37.7 & 41.5 & 15.1 & 3.8 & 1.9 \\
\hline
\end{tabular}

En la Tabla 3 se muestra la representación porcentual del grado de inclusión de ciertos alimentos en el almuerzo. En ella se destaca una mayor prevalencia del consumo del "segundo o plato fuerte" $(91.5 \%)$ en comparación a la sopa o caldo (75.5\%), teniendo una presencia de carne en un $85.5 \%$. Los piqueos y bebidas alcohólicas constituyen una opción minoritaria en el almuerzo.

\section{Tabla 3}

Porcentaje (\%) de estudiantes universitarios que incluyen diversos alimentos habitualmente en el almuerzo

\begin{tabular}{lcc}
\hline \multicolumn{1}{c}{ Ítem } & SI (\%) & NO (\%) \\
\hline Piqueo (maní, queso en trozos, bocaditos, snacks, papas fritas) & 17.0 & 83.0 \\
Sopa o caldo u otro entrante & 75.5 & 24.5 \\
Plato fuerte o segundo & 91.5 & 8.5 \\
Carne, pescado, pollo o mariscos & 85.5 & 14.5 \\
Arroz, fideos o frijoles & 86.2 & 13.8 \\
Verduras o ensalada & 90.6 & 9.4 \\
Pan, bizcochos, torta, queques o tostadas & 20.8 & 79.2 \\
Postre & 43.4 & 56.6 \\
Fruta & 81.1 & 18.9 \\
Bebida alcohólica: cerveza, vino, ron, otros & 4.4 & 95.6 \\
Bebida endulzada (gaseosas, néctares, jugos, chicha morada) & 58.8 & 41.2 \\
\hline
\end{tabular}

\section{Discusión}

El 53.8\% de los encuestados atribuye a "su contenido nutrimental" como el factor más influyente al elegir un alimento para su ingesta, en tanto un $28.3 \%$ lo atribuye a su "sabor" y el 4.8\% lo constituye el "precio". En España las motivaciones 
que tienen los consumidores al elegir un alimento son: conveniencia (15\%), placer $(14 \%)$, sabor $(12 \%)$, salud $(10 \%)$ y costumbre $(5 \%)$; en tanto el $44 \%$ restante por una combinación de los anteriores (Ministerio de Agricultura, Pesca y Alimentación [MAPA], 2015).

Conocer el aporte nutricional de los alimentos, nos sirve para limitar la ingesta de ciertos nutrientes que, consumidos en demasía pueden ser contraproducentes para nuestra salud, así como un elemento clave para aconsejar y guiar a los pacientes sobre la elección de alimentos saludables y que así puedan seguir indicaciones médicas de manera correcta (Karavaski, 2020).

En cuanto al hábito de leer e interpretar las etiquetas nutrimentales se determinó que un $33 \%$ de los encuestados no las lee por "falta de tiempo", un $22.6 \%$ por "pereza"; en tanto hay un $36.8 \%$ si las lee y las entiende. Una encuesta realizada en Israel entre 1999-2001 a personas comprendidas entre 25-64 años revela que el $83.5 \%$ de los encuestados comprendía bien o muy bien el etiquetado nutricional (Karavaski, 2020). Se ha determinado que aquellas personas que consideran de importancia la salud y la alimentación saludable son aquellas que leen el etiquetado nutricional, lo cual les permite elegir alimentos más saludables (Drichouti et al., 2005) consumiendo alimentos con bajo contenido graso y un mayor consumo de frutas y vegetales (Neuhouser et al., 1999).

Entre el motivo por el cual se evita el consumo de un alimento se destaca que $46.2 \%$ lo hace "porque no le gusta", en tanto un $31.1 \%$ por "cuidarse". La bibliografía establece que la preferencia o aversión a un determinado alimento se debería en parte a la herencia cultural (Bayés, 1983), a la asociatividad entre el consumo de un alimento y la experimentación con algún tipo de enfermedad gastrointestinal, a la asociación del olor con medicamentos que generan alteraciones gastrointestinales (Schafe y Bernstein, 1996), así como al simbolismo del alimento (creencias sobre el origen o la calidad) (Logue et al., 1981).

Un 45.3\% de los encuestados prepara mayoritariamente los alimentos al "vapor o hervidos", un $38.7 \%$ en "guisados o salteados", en tanto un $13.2 \%$ mediante "frituras". Las ventajas de los alimentos cocidos al vapor es que las sustancias hidrosolubles se retienen mejor y el alimento resulta más sápido, por lo que se preservan mejor sus cualidades organolépticas y nutricionales (García, 2008 y Gil et al., 2017).

El $46.2 \%$ de los encuestados manifiestan que es la madre la que prepara los alimentos durante la semana, en tanto un $31.1 \%$ de los estudiantes establece que ellos mismos se preparan sus alimentos. El elaborar los propios alimentos, permitirá conocer sobre ellos, cuales presentan mayor contenido en nutrientes, fomenta asimismo una mayor conciencia de lo que se ingiere; por otra parte, es más fácil dosificar las raciones que uno consume sin quedarse con hambre, evitando el riesgo de comer en exceso (Pérez, 2018).

En cuanto a la actitud que toma el encuestado cuando se siente satisfecho después de las comidas: Un 78.9\% considera que "deja de comer sin problemas"; en tanto hay un $12.9 \%$ que sigue comiendo sin problema. Muchas personas desconocen 
el tamaño que debe tener las raciones para considerarlas "adecuadas" y por consecuencia ingieren un exceso de energía (Stubbs et al., 1996).

En cuanto a la frecuencia al masticar los alimentos se estableció la pregunta si aprueba considerar masticar más de 25 veces cada bocado de comida. Un 33.9\% está de "acuerdo" y "totalmente de acuerdo". La masticación es un proceso fisiológico controlado por el sistema nervioso central y modulado por impulsos de la boca. La edad, el sexo y el estado dentario constituyen los factores intrínsecos más estudiados, mientras que la dureza, características reológicas como plasticidad o elasticidad y tamaño de los alimentos son los factores extrínsecos más conocidos (Woda et al., 2006).

Establecer un número concreto de masticación es muy rígido debido a que cada alimento requiere una masticación distinta para poder triturarse, lo que si queda establecido es que a mayores recuentos de masticación se reduce la ingesta de alimentos (Smit et al., 2011). Paphangkorakit et al. (2019) evaluaron el consumo de un almuerzo habitual en jóvenes entre 18 y 24 años, determinando que el número de masticación por bocado estaría entre 19,2 $\pm 6,4$. Por otra parte ingerir alimentos pausadamente incrementa los niveles de colecistoquinina, hormona relacionada con la saciedad, así como disminuye la concentración de grelina, hormona que promueve el apetito en el cerebro (Milke,2005).

En cuanto al número de veces que consumen los alimentos durante la semana y fin de semana. Un $66 \%$ de los encuestados consume tres veces los alimentos tanto entre semana como los fines de semana. Solo un $20.8 \%$ consume cinco comidas al día (lunes-viernes) y un 7.5\% lo hace los sábados y domingos. El porcentaje de estudiantes que consumen cinco comidas al día entre semana es mayor que los fines de semana. Carbajal (2013) considera que la ingesta de cinco comidas al día es saludable y que tres ingestas diarias podrían favorecer a mantener los niveles de glucosa bajos, especialmente para personas con diabetes. Aunque ello dependerá de edad de la persona, actividad que realice, estados de salud, entre otras.

Las preferencias de ciertos alimentos (Tabla 2) en la categoría "me agrada mucho" y "me agrada" en conjunto suman $96.2 \%$ para frutas, $78.3 \%$ para verduras. Sin embargo, las preferencias no coinciden con lo que realmente se consume. Se establece que el $11.3 \%$ de la población mayor de 15 años en el Perú consume cinco porciones de frutas y/o verduras al día. Los departamentos de Ucayali y Pasco presentan el menor consumo ( $4.6 \%$ y $5.5 \%$ respectivamente), mientras que el mayor consumo ocurre en Arequipa, seguido de Lima, Piura, Loreto y La Libertad (15.4\%, 13.8\%, 13.8\%, 13.2\% y $13.1 \%$ de respectivamente) (Domínguez, 2020).

La OMS recomendó en el 2005 un consumo diario de al menos 600 gramos de frutas y hortalizas en adultos (Lock et al., 2005). Existe una preferencia del $85.2 \%$ por la carne y pollo; en tanto para pescados y mariscos llega al 77.4\%. Esto probablemente a que la población de la sierra Liberteña no cuenta con acceso rápido y fresco de los recursos hidrobiológicos que ofrece el mar y que además sus precios son mayores.

Dentro de las bebidas que mayormente consumen los estudiantes durante el día es agua (70.8\%) en comparación a los jugos, néctares o tés industrializados (17\%) y 
a la gaseosa $(8.5 \%)$. Por otra parte, lo que optan los estudiantes entre comidas es de preferencia la fruta y verdura (58.5\%), seguido de papa rellena, salchipollo y snacks (12.3\%) y de galletas, pan, bizcochos, churros (8.5\%) como los más representativos.

En cuanto a las preferencias alimentarias se refiere (Tabla 3) se evidencia que un $75.5 \%$ de los encuestados consume sopa, caldo o entrante en el almuerzo. Dependiendo como este elaborada esta puede constituir un buen hábito alimentario (Márquez et al., 2014). El consumo de sopa se asocia a un menor aporte energético y una mejor calidad en la alimentación en los adultos estadounidenses permitiendo un buen aporte de líquido para su hidratación (Zhu y Hollis, 2014).

Un 20.8\% de los encuestados incluye al pan, bizcochos, tortas, queques o tostadas en sus almuerzos. Hay desacuerdos en cuanto a la presencia de pan en el almuerzo. Por una parte, se considera que no es aconsejable comer pan durante las comidas, puesto que solo incrementa las calorías de la comida principal. Por otra parte, la OMS recomienda un consumo de 200-250 g/día.

Un $43.4 \%$ de los encuestados incluye al postre en sus almuerzos. Se ha establecido que, una vez que terminamos una comida importante como el almuerzo, el organismo genera la insulina necesaria para metabolizarla, y si es que elegimos un alimento con intenso dulzor, estamos promoviendo a nuestro cuerpo a generar más, para administrar glúcidos de rápida absorción, lo que promueve en el tiempo en aumento de azúcar en sangre, diabetes y enfermedades del corazón (Destefano, 2017).

Un 95.6\% de los encuestados no incluye bebidas alcohólicas como cerveza, vino, ron, otros en sus almuerzos. Se ha establecido que el alcohol (etanol), una vez absorbido en el estómago, favorece la vasodilatación, reduce la presión sistólica, reduce los fibrinógenos y aumenta los valores de DHL (lipoproteínas de alta densidad del colesterol) (Ortuño,2009); sin embargo, en cantidades no moderadas durante la comida, el alcohol dificulta la digestión y origina trastornos sobre el tubo digestivo, especialmente de la motilidad esofágica, aparición de gastritis, trastornos del vaciado y diarreas, además de ser un elemento depresor del sistema nervioso central (Téllez y Cote, 2006).

Un 58.8\% de los encuestados incluye bebidas endulzadas como gaseosas, néctares, jugos y chicha morada con azúcar. El peruano consume en promedio al año 19.5 kilos de azúcar. Esta cantidad no toma en cuenta el azúcar con que cuentan los alimentos procesados, con lo cual, el consumo anual per cápita puede llegar a 21 o 22 kilos (Instituto Nacional de Estadística e Informática [INEI], 2012). Las bebidas gasificadas proveen excesivas calorías y un escaso beneficio nutricional (Rodríguez et al., 2014).

Un $22.6 \%$ de los encuestados come de 1-2 veces por semana fuera de casa; un $19.8 \%$ lo hace de 5-6 veces; en tanto un $21.7 \%$ come una vez al mes o menos de una vez por semana. Según NielsenIQ (2016), al menos una vez a la semana el $42 \%$ de los peruanos come fuera de casa, donde el 10\% corresponde al desayuno, seguido de la cena con $51 \%$ y el almuerzo con $70 \%$. 
En cuanto a la percepción de consumir en exceso: un 33\% de los estudiantes encuestados lo hace de 1-2 veces por semana; en tanto un 17.9\% lo hace de 3-4 veces por semana. La valoración porcentual conjunta de aquellos que los hacen "una vez al mes" o "menos de una vez al mes" llega al 23.6\%. Comer en exceso puede causar trastornos en la alimentación, la cual es considerada como una enfermedad, la cual generalmente aparecen durante la adolescencia o adultez temprana. El trastorno por atracón es un tipo de trastorno de la alimentación, además de la anorexia o bulimia y que están asociadas a la depresión, el abuso de sustancias o los trastornos de ansiedad (Steinhausen, 2008).

Entre las acciones que harían falta para mejorar la alimentación: un 39\% de los encuestados considera "compromiso o motivación personal", un $26.4 \%$ "más información". En tanto menos de un 1\% establece que "nada", pues considera que su alimentación es saludable. En cuanto a la percepción del grado de diversidad en el consumo de una dieta alimentaria: un $45.6 \%$ considera que es "diferente solo algunas veces durante la semana"; en tanto un $40.6 \%$ es "diferente cada día". Solo un $5 \%$ de los encuestados considera que recibe una dieta muy monótona.

En cuanto al grado de aceptación de recibir un consejo de nutrición para mejorar el estado de salud: un 48.1\% de los encuestados está "de acuerdo" y un $34.9 \%$ "totalmente de acuerdo". Ambas respuestas son consideradas como indicadores de un buen comportamiento alimentario. Sin embargo, hay un $10.1 \%$ se muestran reacios a recibir consejos relacionados a la nutrición.

Si bien la mayoría de los resultados aquí presentados constituyen aproximaciones a un buen comportamiento alimentario faltarían estudios que permitan especificar las raciones o cantidades que las personas encuestadas consumen. El tener jóvenes conscientes de que sus comportamientos frente a la alimentación van a influir en su salud, su aspecto físico, en sus relaciones sociales y personales constituye una tarea del Estado y la Academia.

\section{Conclusiones}

En términos generales los estudiantes de la UNT-Filial Huamachuco presentan comportamientos alimentarios saludables, destacando el consumo de sopa, caldo $\mathrm{u}$ otro entrante en el almuerzo en un $75.5 \%$, un $91.5 \%$ de un plato fuerte o segundo.

El 53.8\% de los estudiantes universitarios determina como factor de elección de un alimento su contenido nutrimental. Un 33\% no lee las etiquetas nutrimentales por falta de tiempo, en tanto un $36.8 \%$ las lee, pero no las entiende. Un $46.2 \%$ establece que no consume un determinado alimento bajo el criterio "que no le gusta". Un $45.3 \%$ considera al vapor o hervido como el tipo de preparación más habitual de un alimento, siendo la madre en un $46.2 \%$ la persona quien lo realiza.

A partir de los resultados obtenidos se evidencia a nivel universitario la implementación de programas que permitan fortalecer los hábitos alimentarios 
relacionados a la frecuencia y dosificación de alimentos ingeridos, la interpretación de etiquetas nutricionales, así como a la forma de preparación y consumo de los alimentos.

Promover comportamientos alimentarios saludables es contribuir con el bienestar físico, mental y social del individuo, permitiendo reducir los costos de atención sanitaria tanto para el Estado como a las familias con bajos recursos económicos.

\section{Agradecimientos}

Los autores agradecen en la persona de Dra. Yolanda Fabiola Márquez Sandoval y colaboradores las facilidades de compartir su cuestionario y la correspondiente adaptabilidad para esta investigación.

\section{Declaración de conflicto de intereses}

Los autores declaran no tener conflictos de intereses financieros ni personales que puedan influir inapropiadamente en el desarrollo de este artículo. 


\section{Referencias}

Álvarez, G. \& Bendezú, R. (2011). Estado nutricional y su relación con los hábitos alimenticios de los internos de la EAP de Enfermería de la Universidad Wiener, 2011 [Tesis de pregrado, Universidad Wiener-Lima-Perú] http://repositorio. uwiener.edu.pe/xmlui/handle/123456789/44

Bayés, R. (1983). Preferencias y aversiones alimenticias en una población española. Revista de Psicología General y Aplicada, 38,513-528. https://dialnet.unirioja. es/servlet/articulo?codigo $=3006662$

Cabrera, G. \& Galarza, V. (2008). Hábitos alimentarios saludables. Programa subvencionado por el Ministerio de Sanidad y Consumo de España [Archivo PDF]. http://www.aytojaen.es/portal/RecursosWeb/DOCUMENTOS $\underline{/ 1 / 0 \quad 1163 \text { 1.pdf }}$

Carbajal, A. (2013). Manual de Nutrición y Dietética. Universidad Complutense de Madrid -España. https://core.ac.uk/reader/19724283

Caswell, J. \& Padberg, D.(1992). Toward a more comprehensive theory of food labels. American Journal of Agricultural Economics, 74 (2), 460-468.https://doi. org/10.2307/1242500

Chota, C. \& Simon, S. (2020). Factores que determinan los hábitos alimentarios de los estudiantes de la Escuela Profesional de Enfermería de la Universidad Nacional de Ucayali, 2019 [Tesis de grado, Universidad Nacional de Ucayali-Perú]. http://repositorio.unu.edu.pe/handle/UNU/4266

Consejo Consultivo de Radio y Televisión, CONCORTV. (2012). Análisis de la publicidad de alimentos no saludables en la televisión peruana. http:// www.concortv.gob.pe/file/informacion/estudios/2012/concortv-analisispublicidad-alimentos-tv-peru.pdf

Destefano, G. (16 marzo, 2017). La verdad detrás del consumo de postres después del almuerzo. https://www.ultimahora.com/la-verdad-detras-del-consumopostres-despues-del-almuerzo-n1070588.html

Domínguez, C. (11 setiembre, 2020). INS: 11.3\% de peruanos consume las cantidades de frutas y verduras que sugiere la OMS. Diario el Peruano. https://elperuano. pe/noticia/103552-ins-113-de-peruanos-consume-las-cantidades-de-frutasy-verduras-que-sugiere-la-oms

Drichoutis, A., Lazaridis, P. \& Nayga, R. (2005). Nutrition knowledge and consumer use of nutritional foods labels. European Review of Agricultural Economics, 32(1), 93-118. https://doi.org/10.1093/erae/jbi003 
García, V. (2008). Técnicas de cocina. Editorial Ediciones Norma-Capitel.

Gil, A., Juárez, M. \& Fontecha, J. (2017). Influencia de los procesos tecnológicos sobre el valor nutritivo de los alimentos. En: Ángel Gil, editor. Tratado de Nutrición, 3. a ed. Composición y calidad nutritiva de los alimentos. Tomo III:

Panamericana. pp. 585-621.

Huanca, C. \& Valero, J. (2020). Conocimientos sobre alimentación saludable en estudiantes de enfermería de la Universidad María Auxiliadora, 2019 [Tesis de grado, Universidad María Auxiliadora-Lima-Perú]. http://repositorio.uma. edu.pe/handle/UMA/254

Instituto Nacional de Estadística e Informática. (2012). Perú: Consumo per cápita de los principales alimentos 2008-2009. Centro de Edición de la Oficina Técnica de Difusión del INEI [Archivo PDF].https://www.inei.gob.pe/media/Menu Recursivo/publicaciones_digitales/Est/Lib1028/Libro.pdf

Irazusta, A., Hoyos, I., Díaz, E., Irazusta, J., Gil, S. \& Gil, J. (2007). Alimentación de estudiantes universitarios. Osasunaz, 8, 7-18. http://www.euskomedia.org/ PDFAnlt/osasunaz/08/08007018.pdf

Karavaski, N. (2020). La importancia de la correcta interpretación del rotulado nutricional. Revista Fronteras en Medicina, 15(1), 31-35. https://DOI.org/ 10.31954/RFEM/202001/0033-0037

Lock, K., Pomerleau, J., Causer, L., Altmann, D. \& McKee, M. (2005). The global burden of disease attributable to low consumption of fruit and vegetables: implications for the global strategy on diet. Bull World Health Organ, 83(2), 100-108. https://www.ncbi.nlm.nih.gov/pmc/articles/PMC2623811/ pdf/15744402.pdf

Logue, A., Ophir I. \& Strauss, E. (1981). The acquisition of taste aversions inhumans. Behavioral Research and Therapy, 19,319-333. https://doi.org/10.1016 10005-7967(81)90053-X

Mariño, G. Núñez, V. \& Gámez, B. (2016). Alimentación saludable. Revista Acta Médica, 17(1).https://www.medigraphic.com/pdfs/actamedica/acm-2016/ acm161e.pdf

Márquez Sandoval, Y., Salazar Ruiz, E., Macedo Ojeda, G., Altamirano Martínez, M., Bernal Orozco, M., Salas Salvadó, J. \& Vizmanos Lamotte, B. (2014). Diseño y validación de un cuestionario para evaluar el comportamiento alimentario en estudiantes mexicanos del área de la salud. Nutrición Hospitalaria, 30(1), 153-164. https://dx.doi.org/10.3305/nh.2014.30.1.7451 
Milke, G. (2005). Ghrelina: más allá de la regulación del hambre. Revista de Gastroenterología de México, 70 (4), 465-473. https://www.medigraphic. com/pdfs/gastro/ge-2005/ge054m.pdf

Ministerio de Agricultura, Pesca y Alimentación (2015). Panel de usos de los alimentos.

[Archivo PDF] https://www.mapa.gob.es/es/pac/post-2020/20200622 oe91 documentopartidav7 tcm30-520580.pdf

Neuhouser, M., Kristal, A. \& Patterson, R. (1999). Use of food nutrition labels is associated with lower fat intake. Joumal of the American Dietetic Association, 99(1),45-53. https://doi.org/10.1016/S0002-8223(99)00013-9

NielsenIQ (2016). 42\% de los peruanos come fuera de su hogar al menos una vez a la semana. https://nielseniq.com/global/es/insights/analysis/2016/42-por-cientode-los-peruanos-come-fuera-de-su-hogar-al-menos-una-vez-a-la-semana/

Organización Mundial de la Salud. OMS. (1998). Recommended nutrient reference values for food labelling purposes.Report of a Joint FAO/WHO Expert Consultation on recommended allowances of nutrients for food labelling purposes. Helsinki, Finland.

Organización Mundial de la Salud. OMS. (13 de abril 2021). Enfermedades no transmisibles.https://www.who.int/es/news-room/fact-sheets/detail/ noncommunicable-diseases

Ortuño, G. (2009). Salud y consumo moderado de vino. Revista Electrónica Enfermería Global, 15,1-15. https://scielo.isciii.es/pdf/eg/n15/miscelanea2.pdf

Paphangkorakit, J., Kanpittaya, K., Pawanja, N. \& Pitiphat, W. (2019). Effect of chewing rate on meal intake. The European Journal of Oral Sciences, 127(1), 40-44. https://doi.org/10.1111/eos.12583

Pérez, R. (2018). Dejar de cocinar nos está matando: siete beneficios de hacerte tu propia comida. https://www.vitonica.com/wellness/dejar-de-cocinar-nosesta-matando-7-beneficios-de-hacerte-tu-propia-comida

Rodríguez, M., Avalos, M. \& López, C. (2014). Consumo de bebidas de alto contenido calórico en México: un reto para la salud pública. Salud en Tabasco, 20 (1), 28-33. https://www.redalyc.org/pdf/487/48731722006.pdf

Ruiz, M. (2019). Conocimientos, actitudes y prácticas sobre alimentación saludable en adolescentes de la institución educativa Juan Ingunza Valdivia, Callao 2018 [Tesis de grado, Universidad Nacional Federico Villareal, Lima-Perú] http:// repositorio.unfv.edu.pe/handle/UNFV/3358 nih.gov/8862476/ 
Schafe, G. \& Bemstein, I. (1996). Taste aversion learning. En E.D.Capaldi (Ed.), Why we eat what we eat. The psychology of eating (pp. 31-51). American Psychological Association. https://doi.org/10.1037/10291-002

Smit, H., Kemsley, E., Tapp, H. \& Henry, C. (2011). Does prolonged chewing reduce food intake? Fletcherism revisited. Appetite, 57(1),295-298. https://doi.org/ $\underline{10.1016 / j . a p p e t .2011 .02 .003}$

Steinhausen, H. (2008). Outcomes of eating disorders. Child and Adolescent Psychiatric Clinics of North America, 18, 225-242. https://doi.org/10.1016/ j.chc.2008.07.013

Stubbs, R., van Wyk, M., Johnstone, A. \& Harbron, C. (1996). Breakfasts high in protein, fat or carbohydrate: effect on within-day appetite and energy balance. European Journal of Clinical Nutrition, 50, 409-417. https://pubmed.ncbi.nlm. .nih.gov/8862476/

Téllez, J. \& Cote, M. (2006). Alcohol etílico: Un tóxico de alto riesgo para la salud humana socialmente aceptado. Revista de la Facultad de Medicina Universidad Nacional de Colombia, 54, (1), 33-47 http://www.scielo.org.co/ pdf/rfmun/v54n1/v54n1a05.pdf

Valero, J. (2020). Conocimientos sobre alimentación saludable en estudiantes de enfermería de la Universidad María Auxiliadora, 2019 [Tesis de grado, Universidad María Auxiliadora-Lima-Perú]. http://repositorio.uma.edu.pe/ $\underline{\text { handle/UMA/254 }}$

Woda, A., Foster, K., Mishellany, A. \& Peyron, M. (2006). Adaptation of healthy mastication to factors pertaining to the individual or to the food. Physiology \& Behavior, 30, 89(1), 28-35. https://doi.org/10.1016/j.physbeh.2006.02.013

Zhu, Y. \& Hollis, J. (2014). Soup consumption is associated with a lower dietary energy density and a better diet quality in US adults. British Journal of Nutrition, 111(8), 1474-1480. https://doi.org/10.1017/S0007114513003954 


\section{ANEXOS \\ Cuestionario del comportamiento del alimento (Márquez-Sandoval et al., 2014-adaptado)}

1. ¿Qué factor consideras más importante al elegir un alimento para su consumo?
a. Su sabor
b. Su precio
c. Que sea agradable a la vista
d. Su caducidad
e. Su contenido nutrimental

2. Me es difícil leer las etiquetas nutrimentales:
a. Por falta de tiempo
b. Porque no me interesa
c. Porque no las entiendo
d. Por pereza
e. Sí las leo y las entiendo

3. Si evitas algún alimento, ¿Por qué motivo lo haces?

$\square$ a. Porque no me gusta

b. Por cuidarme

c. Porque me hace sentir mal

d. No suelo evitar ningún alimento

4. ¿Cuál es la preparación más habitual de tus alimentos?
b. Al vapor o hervidos
c. Asados o a la plancha
d. Horneados
e. Guisados o salteados

a. Fritos (Incluye empanizados y capeados)

5. ¿Quién prepara tus alimentos con mayor frecuencia durante la semana?
a. Yo
b. Mi mamá
c. Los compro ya preparados
d. Otro:

6. ¿Qué haces normalmente cuando te sientes satisfecho?
a. Dejo de comer sin problema
b. Dejo de comer, pero me cuesta hacerlo
c. Sigo comiendo sin problema
d. Sigo comiendo, pero me siento mal de hacerlo 
7. ¿Qué haces con la grasa visible de la carne?
a. La quito toda
b. Quito la mayoría
c. Quito un poco
d. No quito nada

8. Habitualmente mastico cada bocado más de 25 veces:
a. Totalmente en desacuerdo
b. En desacuerdo
c. Ni de acuerdo ni en desacuerdo
d. De acuerdo
e. Totalmente de acuerdo

9. Marque las veces con las que habitualmente ingieres alimentos durante el día

\begin{tabular}{|c|c|}
\hline Entre semana (lunes-viernes) & $\begin{array}{c}\text { El fin de semana } \\
\text { (sábado-domingo) }\end{array}$ \\
\hline 2 veces por día ( & 2 veces por día $(\quad)$ \\
\hline 3 veces por día ( & 3 veces por día ( \\
\hline 4 veces por día ( & 4 veces por día ( \\
\hline 5 veces por día ( & 5 veces por día ( \\
\hline
\end{tabular}

Marca la opción correspondiente de acuerdo a tu agrado para comer los siguientes alimentos

\begin{tabular}{r|l|c|c|c|c|c}
\hline $\mathrm{N}^{\circ}$ & \multicolumn{1}{|c|}{ Alimento } & $\begin{array}{c}\text { a. Me } \\
\text { agrada } \\
\text { mucho }\end{array}$ & $\begin{array}{c}\text { b. Me } \\
\text { agrada }\end{array}$ & $\begin{array}{c}\text { c. Ni me } \\
\text { agrada ni } \\
\text { me } \\
\text { desagrada }\end{array}$ & $\begin{array}{c}\text { d. Me } \\
\text { desagrada }\end{array}$ & $\begin{array}{c}\text { e. Me } \\
\text { desagrad } \\
\text { a mucho }\end{array}$ \\
\hline 10. & Frutas & $\square$ & $\square$ & $\square$ & $\square$ & $\square$ \\
11. & Verduras & $\square$ & $\square$ & $\square$ & $\square$ & $\square$ \\
12. & Carnes y pollo & $\square$ & $\square$ & $\square$ & $\square$ & $\square$ \\
13. & Pescados y mariscos & $\square$ & $\square$ & $\square$ & $\square$ & $\square$ \\
14. & Lácteos & $\square$ & $\square$ & $\square$ & $\square$ & $\square$ \\
15. & $\begin{array}{l}\text { Pan, papa, fideos, } \\
\text { cereales }\end{array}$ & $\square$ & $\square$ & $\square$ & $\square$ \\
16. & $\begin{array}{l}\text { Frijoles, garbanzos, } \\
\text { lentejas }\end{array}$ & $\square$ & $\square$ & $\square$ & $\square$ & $\square$ \\
17. & Huevo & $\square$ & $\square$ & $\square$ & $\square$ & $\square$ \\
18. & $\begin{array}{l}\text { Almendras, nueces, } \\
\text { pasas, guindones, etc. }\end{array}$ & $\square$ & $\square$ & $\square$ & $\square$ & $\square$ \\
19. & Bebidas alcohólicas & $\square$ & $\square$ & $\square$ & $\square$ & $\square$ \\
20. & Alimentos envasados & $\square$ & $\square$ & $\square$ & $\square$ & $\square$ \\
\hline
\end{tabular}


21. ¿Qué sueles beber en mayor cantidad durante el día?
a. Gaseosa
b. Agua
c. Jugos, néctares o tés industrializados
d. Leche
e. Otro:

22. ¿Qué sueles ingerir habitualmente entre comidas?

$\square$ a. Dulces

b. Fruta o verdura

c. Galletas, pan, bizcochos, churros

d. Yogurt

e. Papa rellena, salchipollos, snacks, frituras, etc.

f. Maní, chochos, pasas u otras semillas.

$\square$ g. Nada

23. ¿Qué incluyes habitualmente en tu almuerzo?

\begin{tabular}{llll}
\hline $\mathrm{N}^{\circ}$ & Alimento & a. & b \\
23.1 & Piqueo (maní, queso en trozos, bocaditos, snacks, papas & sí $\square$ & no \\
& fritas) & sí $\square$ & no $\square$ \\
23.2 & Sopa o caldo u otro entrante & sí $\square$ & no $\square$ \\
23.3 & Plato fuerte o segundo & sí $\square$ & no $\square$ \\
23.4 & Carne, pescado, pollo o mariscos & sí $\square$ & no $\square$ \\
23.5 & Arroz, fideos o frijoles & sí $\square$ & no $\square$ \\
23.6 & Verduras o ensalada & sí $\square$ & no $\square$ \\
23.7 & Pan, bizcochos, torta, queques o tostadas & sí $\square$ & no $\square$ \\
23.8 & Postre & sí $\square$ & no $\square$ \\
23.9 & Fruta & sí $\square$ & no $\square$ \\
23.10 & Bebida alcohólica: cerveza, vino, ron, otros & sí $\square$ & no $\square$ \\
23.11 & Bebida endulzada (gaseosas, néctares, jugos, chicha & & \\
& morada) & & \\
\hline
\end{tabular}

24. ¿Con que frecuencia comes alimentos fuera de casa?
a. Todos los días
b. 5 a 6 veces a la semana
c. 3 a 4 veces a la semana
d. 1 a 2 veces a la semana
e. Una vez cada 15 días
f. Una vez al mes
g. Menos de una vez al mes 
25. ¿Con qué frecuencia crees que comes alimentos en exceso?

$\square$ a. Todos los días

b. 5 a 6 veces a la semana

c. 3 a 4 veces a la semana

d. 1 a 2 veces a la semana

e. Una vez cada 15 días

f. Una vez al mes

g. Menos de una vez al mes

26. ¿Qué haces o estarías dispuesto a hacer para cuidar tu cuerpo?

$\checkmark$ a. Cuidar mi alimentación

b. Seguir un régimen dietético temporal

c. Hacer ejercicio

d. Cuidar mi alimentación y hacer ejercicio

e. Tomar suplementos dietéticos o productos herbolarios

f. Nada

27. ¿Qué consideras que te hace falta para mejorar tu alimentación?

$\square$ a. Más información

b. Apoyo social

c. Dinero

d. Compromiso o motivación personal

e. Tiempo

f. Nada, creo que mi alimentación es saludable

$\square$ g. No me interesa mejorar mi alimentación

28. Consideras que tu dieta alimentaria (lo que comes) es:

a. Diferente cada día

b. Diferente solo algunas veces durante la semana

c. Diferente solo durante los fines de semana

d. Muy monótona

29. ¿Crees que eres capaz de utilizar un consejo de nutrición para mejorar tu estado de salud?

$\square$ a. Totalmente en desacuerdo

b. En desacuerdo

c. Ni de acuerdo ni en desacuerdo

d. De acuerdo

e. Totalmente de acuerdo

Nota: La(s) alternativa(s) en negrita son consideradas como respuestas saludables. 